\title{
Can déjà vu result from similarity to a prior experience? Support for the similarity hypothesis of déjà vu
}

\author{
Anne M. Cleary, Anthony J. Ryals, and Jason S. Nomi \\ Colorado State University, Fort Collins, Colorado
}

\begin{abstract}
The strange feeling of having been somewhere or done something before-even though there is evidence to the contrary-is called déjà $v u$. Although déjà vu is beginning to receive attention among scientists (Brown, 2003, 2004), few studies have empirically investigated the phenomenon. We investigated the hypothesis that déjà vu is related to feelings of familiarity and that it can result from similarity between a novel scene and that of a scene experienced in one's past. We used a variation of the recognition-without-recall method of studying familiarity (Cleary, 2004) to examine instances in which participants failed to recall a studied scene in response to a configurally similar novel test scene. In such instances, resemblance to a previously viewed scene increased both feelings of familiarity and of déjà vu. Furthermore, in the absence of recall, resemblance of a novel scene to a previously viewed scene increased the probability of a reported déjà vu state for the novel scene, and feelings of familiarity with a novel scene were directly related to feelings of being in a déjà vu state.
\end{abstract}

Most people have experienced déjà vu at some point. Déjà $v u$ is the odd feeling of having experienced a situation before, despite the fact that the situation is new (e.g., Brown, 2004). One theory is that déjà vu is related to feelings of familiarity (Brown, 2003, 2004; Cleary, 2008). In the present study, we examined the hypothesis that, when a novel scene is similar to a previously viewed scene, it can produce a feeling of familiarity that is related to déjà vu.

\section{Déjà Vu and Familiarity}

Familiarity-based recognition occurs when one has the feeling that something has been experienced before, despite not recalling any specifics about the prior experience (e.g., Mandler, 2008). An example is recognizing a face as familiar without identifying where or when the face was seen before. The feeling of familiarity that underlies familiarity-based recognition may also contribute to experiences of déjà vu (Cleary, 2008).

\section{Is Déjà Vu Related to Familiarity?}

There are three lines of evidence that déjà vu may be related to familiarity. One line comes from correlational data. The frequency with which people report experiencing déjà vu is positively correlated with the frequency with which people report traveling (Brown, 2003), dreaming (Brown, 2003), and watching movies (Wallisch, 2007). These correlations are consistent with the idea that déjà vu relates to familiarity: People who travel, dream, or watch movies more often should have more potential sources of familiarity stored in memory than people who rarely do these things.
The second line of evidence comes from a study by Brown and Marsh (2008), in which students viewed pictures of scenes - some from the students' own campus and some from a faraway campus. The students later attempted to discriminate previously visited from non-previously visited scenes. They were more likely to judge that they had previously visited a scene from a faraway campus when that scene was previously presented within the context of the experiment. In short, when the feeling of familiarity was unaccompanied by retrieval of the familiarity's source, it led to a sense of having been there before. This hints at a potential link between familiarity and déjà vu.

The third line of evidence comes from a case study of a patient who, prior to surgical intervention, experienced déjà vu during seizures (Bowles et al., 2007). Although the surgical intervention relieved the patient of seizures, she was subsequently impaired in familiarity-based, but not recollection-based, recognition on a number of recognition tasks. This postsurgery impairment, along with the seizure-induced déjà vu that she experienced prior to surgery, also suggests a possible relationship between familiarity-based recognition and déjà vu.

\section{What Produces Familiarity With a \\ Novel Situation? The Role of Resemblance}

Familiarity with a novel situation can be brought on by resemblance to a situation stored in memory. For example, Cleary (2004) presented participants with a study list of words (e.g., obstetrician, bushel), followed by a test of the participants' ability to discriminate novel test cues that resembled studied items (e.g., obstruction, bashful) from

A. M. Cleary, anne.cleary@colostate.edu 
novel test cues that did not. It was shown that, even when participants could not use a test cue (e.g., obstruction) to recall a studied item that resembled it (e.g., obstetrician), they still gave it a higher recognition rating, on average, when it resembled a studied word than when it did not. In short, participants found novel cues that resembled studied words to be more familiar than novel cues that did not resemble studied words, despite their being unable to recall the specific study episodes responsible for the increased familiarity. This finding is referred to as recognition without cued recall (RWCR).

\section{What Kind of Resemblance Might Produce Déjà Vu?}

One way in which a novel situation might resemble a prior situation in memory to produce déjà vu is in the configuration of elements (Brown, 2004). For example, one might encounter a never-before-seen room and experience déjà vu because the configuration of elements (e.g., the placement of the couches and lamps relative to a painting on the wall) is similar to the configuration of a room seen in one's past. If one knows that the current room is novel, yet cannot identify the prior experience responsible for the familiarity with it, the feeling of familiarity may be labeled as déjà vu.

\section{Empirically Investigating the Similarity Hypothesis of Déjà Vu}

In the present study, we used a scene variation of the RWCR procedure (Cleary, 2004) to investigate the similarity hypothesis of déjà vu. This method is ideally suited for investigating déjà vu for a number of reasons. Whereas traditional studies of familiarity-based recognition require discrimination between actually studied and unstudied items, the RWCR method involves presenting only novel (unstudied) items at test. Thus, the method can potentially mimic the real-life experience of sensing familiarity with a never-before-seen scenario (as in déjà vu). Furthermore, the method can be applied to scenes, the most common elicitor of déjà vu (Brown, 2004), and used to investigate the aforementioned configuration hypothesis through the use of configural resemblance at test. Specifically, it can be used to examine whether, in the absence of recall, familiarity is greater for scenes configurally resembling earlier presented scenes than for scenes not resembling earlier presented scenes, ${ }^{1}$ and whether this holds for the probability or intensity of a déjà vu state as well. Doing so would additionally contribute to a growing body of work on the nature of the features that can produce familiarity (e.g., Cleary, 2004), by addressing whether configural resemblance to a prior experience can produce familiarity-based recognition. It would also allow us to examine whether the feelings of familiarity that presumably underlie RWCR are related to feelings of déjà vu.

Additionally, because the RWCR method separates familiarity judgments that are accompanied from those that are unaccompanied by recall, this task allowed us to examine the hypothesis that participants would differentiate déjà vu from familiarity on the basis of the presence or absence of recall. Although familiarity ratings tend to be higher when judgments are accompanied by recall than when they are not (Cleary, 2004), it is conceivable that participants might differentiate déjà vu from familiarity on the basis of the mystery surrounding the lack of an ability to recall specifics (Cleary, 2008).

\section{EXPERIMENT 1}

In Experiment 1, we searched for a scene RWCR effect (Cleary, 2004) - that is, an ability to discriminate test scenes that resembled studied scenes from test scenes that did not in the absence of an ability to recall the studied scenes in question. We also examined whether this effect was related to déjà vu reports.

\section{Method}

Participants. Seventy-four Colorado State University undergraduates participated in exchange for course credit.

Materials. We created 120 black-and-white scenes for use as potential studied scenes (SSs). For each of these scenes, ${ }^{2}$ a corresponding configurally similar scene (CSS) was created for use at test (for scene examples, see Figure 1). Stimuli ranged from $500 \times$ 500 pixels to $500 \times 600$ pixels. Each SS appeared with its name in the upper left-hand corner.

Procedure. For each participant, $60 \mathrm{SSs}$ were randomly selected from the pool of 120 possible SSs to appear at study. All 120 CSSs appeared at test; thus, half of the test scenes resembled studied items and half did not. Participants completed four study-test blocks. Participants were told beforehand that all test items would be unstudied and that some would resemble studied items and some would not. Within each block, 15 randomly ordered, named SSs (e.g., bathroom) were presented at participant pace at study $(M=5.51 \mathrm{sec} /$ item), and 30 randomly ordered new scenes (CSSs) were presented at test; 15 of these resembled studied scenes and 15 did not. Each test scene appeared for $4 \mathrm{sec}$, after which participants were prompted to attempt to identify a similar studied scene by typing the studied scene's name (e.g., bathroom). After pressing Enter, participants were prompted to rate the familiarity of the test scene using a scale of 0 (very unfamiliar) to 10 (very familiar). Participants were reminded in the instructions that each test list was specific to the immediately preceding study list. Then, participants were asked to indicate with a yes-no response whether or not they were in a déjà vu state. We defined déjà $v u$ as

experiencing a vivid feeling that you have previously experienced something, even when you know you haven't. For example, you may enter a room and experience déjà vu, such that you feel like you have entered or seen that particular room sometime in the past, even when you know that you have never been there before.

After the déjà vu question, participants were given a second chance at identification and were encouraged to guess.

Data were coded by hand to ensure that any misspelled responses or responses that differed from studied names - but would otherwise correctly identify the corresponding studied scene (e.g., "restroom" instead of "bathroom") - were classified as "identified."

\section{Results and Discussion}

Above-chance cued recall of studied counterpart scenes occurred in all experiments reported here (see Table 1). Identification rates for unstudied counterpart scenes reflect the probability of correctly guessing the target scenes' names.

The data of primary interest are the familiarity ratings given to the test scenes for which recall of corresponding 

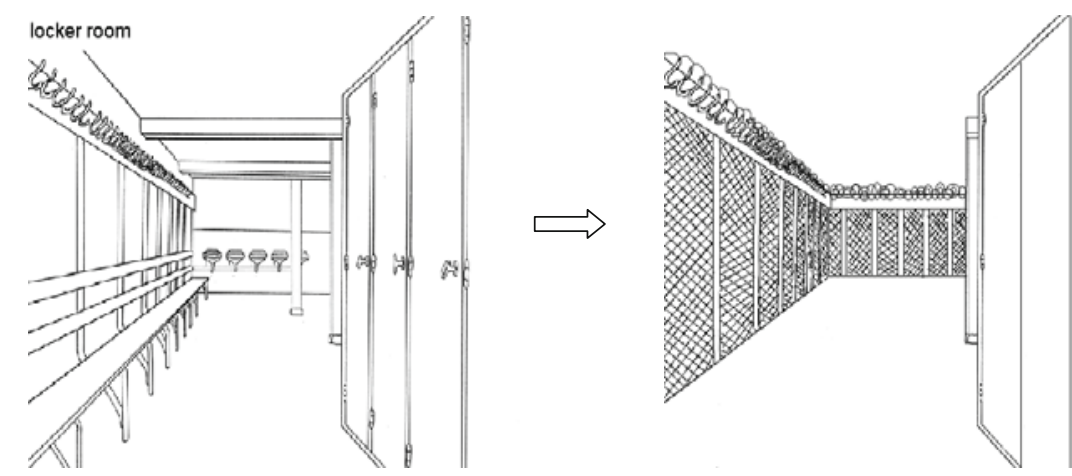

landing strip
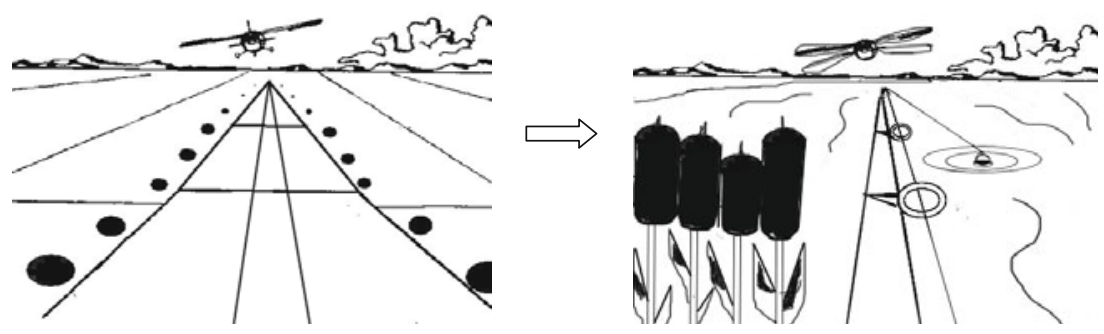

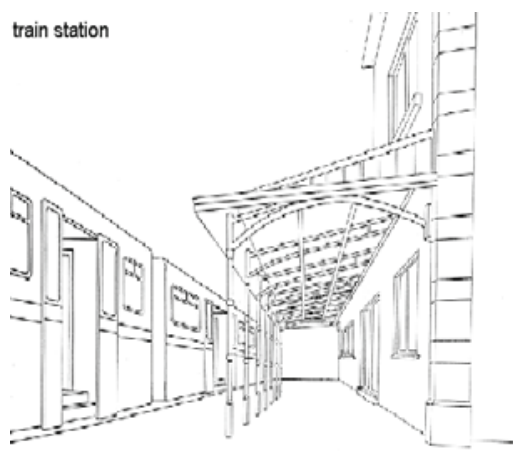

Possible Study Scene
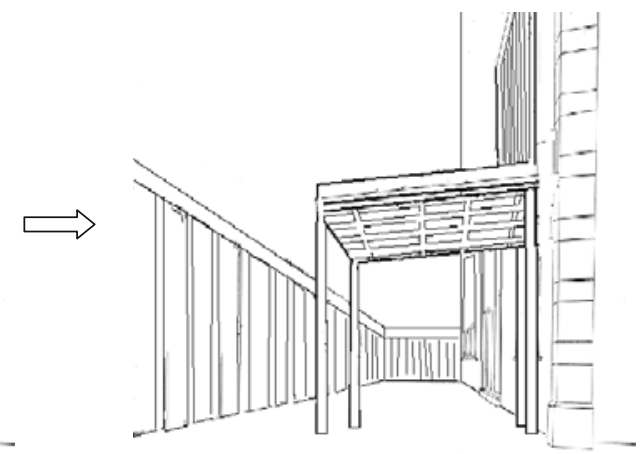

Configurally Similar Test Scene

Figure 1. Examples of possible named study scenes (left column) and their corresponding configurally similar novel test scenes (right column).

Table 1

Mean Cued Recall Rates and Rates of Correctly Guessing Target Unstudied Scenes

\begin{tabular}{|c|c|c|c|c|c|}
\hline \multirow[b]{3}{*}{ Experiment } & \multicolumn{4}{|c|}{ Test Cue Condition } & \\
\hline & \multicolumn{2}{|c|}{$\begin{array}{c}\text { Configurally } \\
\text { Similar } \\
\text { Scene } \\
\text { Studied }\end{array}$} & \multicolumn{2}{|c|}{$\begin{array}{c}\text { Configurally } \\
\text { Similar } \\
\text { Scene } \\
\text { Unstudied }\end{array}$} & \\
\hline & $M$ & $S D$ & $M$ & $S D$ & \\
\hline 1 & .40 & .15 & .02 & .02 & $t(73)=22.58, S E=.02, p<.001$ \\
\hline $2 \mathrm{~A}$ & .43 & .14 & .03 & .03 & $t(49)=19.63, S E=.02, p<.001$ \\
\hline 2B (Familiarity condition) & .46 & .15 & .00 & .00 & $t(46)=20.94, S E=.02, p<.001$ \\
\hline 2B (Déjà vu condition) & .42 & .15 & .00 & .00 & $t(46)=18.70, S E=.02, p<.001$ \\
\hline
\end{tabular}


target scenes did not occur. As is shown in Table 2, ratings were higher for test scenes whose configurally similar counterparts had been studied $\left[t_{\text {subjects }}(73)=5.36, S E=\right.$ $0.06, p<.001, d=0.63]$. This effect persisted in an items analysis based on the 120 test scenes $\left[t_{\text {items }}(119)=4.40\right.$, $S E=0.08, p<.001, d=0.40]$. In short, an RWCR effect (Cleary, 2004) was shown in a situation where the primary resemblance from study to test was in the configuration of elements within a scene. ${ }^{3}$ As is common with this methodology, participants were generally very conservative with their ratings (using the low end of the scale). Note that the RWCR effect refers to the level of similar-dissimilar discrimination, irrespective of where on the scale that discrimination occurred. The magnitude of the RWCR effect did not differ across blocks $(F<1)$; however, participants became less conservative across blocks, so that they gave higher familiarity ratings overall as the experiment progressed $\left[F(3,216)=7.88, M S_{\mathrm{e}}=1.19, p<.001\right]$. Finally, among test items resembling studied items, familiarity ratings were higher in the presence of recall $(M=6.40)$ than in its absence $(M=2.30)[t(73)=25.98, S E=0.16$, $p<.001]$.

We next examined whether the familiarity-underlying scene RWCR might relate to déjà vu. First, among test scenes that did not elicit cued recall, there was an increased probability of a reported déjà vu state for those that resembled studied scenes (see Figure 2$)\left[t_{\text {subjects }}(73)=3.98\right.$, $S E=0.008, p<.001, d=0.47 ; t_{\text {items }}(119)=3.61, S E=$ $0.01, p<.001, d=0.33]$. As with the RWCR effect itself, the magnitude of this effect did not differ significantly across blocks; however, participants became less conservative across blocks, so that they were more likely to report experiencing déjà vu as the experiment progressed $\left[F(3,219)=10.31, M S_{\mathrm{e}}=0.02, p<.001\right]$. As with famil-

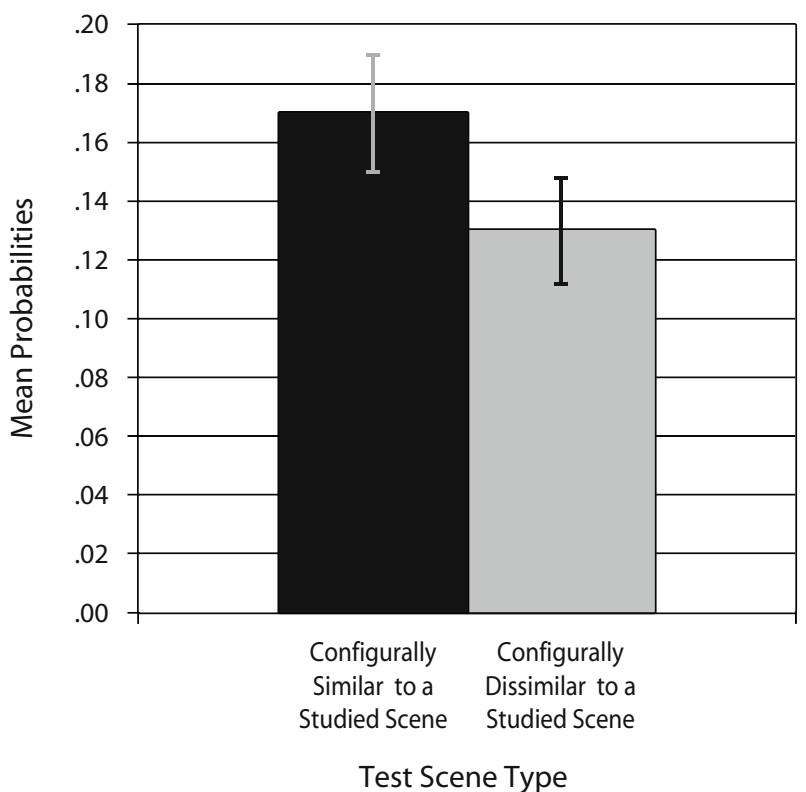

Figure 2. In the absence of cued recall, configural resemblance to a studied scene increased the probability of a déjà vu state for a given novel test scene.

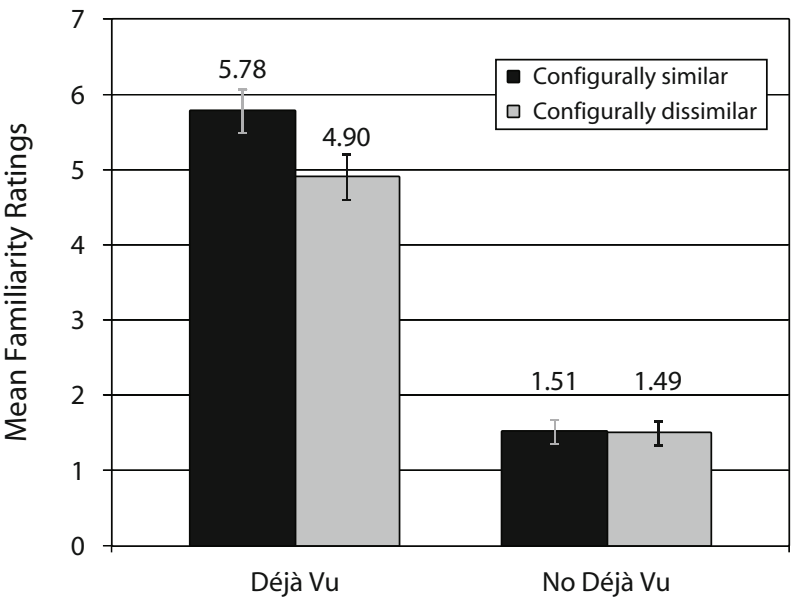

Figure 3. In the absence of cued recall, higher scene familiarity ratings were associated with the presence of déjà vu states, and lower familiarity ratings were associated with the absence of déjà vu states.

iarity ratings, among test items resembling studied items, the probability of reporting déjà vu was higher in the presence of recall $(M=.46)$ than in its absence $(M=.17)$ $[t(73)=9.09, S E=0.03, p<.001]$.

Second, when we treated déjà vu state status as an independent variable in order to conditionalize the familiarity ratings given in the absence of recall on the presence or absence of a reported déjà vu state, ${ }^{4}$ familiarity ratings appeared to be directly related to déjà vu (see Figure 3 ). A $2 \times 2$ déjà vu state (yes vs. no) $\times$ similarity condition (similar vs. dissimilar to a studied scene) repeated measures ANOVA revealed that familiarity ratings were higher for test scenes that elicited déjà vu states than for test scenes that did not $\left[F_{\text {subjects }}(1,51)=297.06, M S_{\mathrm{e}}=2.59, p<.001\right.$, $\eta_{\mathrm{p}}^{2}=.85 ; F_{\text {items }}(1,115)=1,489.28, M S_{\mathrm{e}}=1.15, p<.001$, $\left.\eta_{\mathrm{p}}^{2}=.93\right]$. This suggests a direct correspondence between déjà vu and familiarity. This analysis also revealed that the RWCR effect, shown here as a main effect of study status $\left[F_{\text {subjects }}(1,51)=14.18, M S_{\mathrm{e}}=0.80, p<.001 \eta_{\mathrm{p}}^{2}=.22\right.$; $\left.F_{\text {items }}(1,115)=12.32, M S_{\mathrm{e}}=1.47, p=.001, \eta_{\mathrm{p}}^{2}=.10\right]$ was carried primarily by the ratings given during déjà vu states, as shown by an interaction $\left[F_{\text {subjects }}(1,51)=9.47, M S_{\mathrm{e}}=\right.$ $0.73, p=.001, \eta_{\mathrm{p}}^{2}=.20 ; F_{\text {items }}(1,115)=4.36, M S_{\mathrm{e}}=1.72$, $\left.p=.039, \eta_{\mathrm{p}}^{2}=.04\right]$.

\section{EXPERIMENTS 2A AND 2B}

The results of Experiment 1 suggest that the feeling of familiarity brought on by a given test scene was directly related to the feeling of being in a déjà vu state with the scene. A potential concern regarding Experiment 1, however, is that by juxtaposing the familiarity and déjà vu questions at test, we forced a link between the two. If so, the correspondence between reported feelings of familiarity and reported déjà vu states may simply reflect demand characteristics. Experiments $2 \mathrm{~A}$ and $2 \mathrm{~B}$ examined whether familiarity and déjà vu are still associated when the two are not juxtaposed within the experiment. 
In Experiment 2A, we examined whether similarity to a studied scene would still increase the probability of a reported déjà vu state in the absence of cued recall when there was no reference to familiarity and participants were given no definition of déjà vu. In Experiment 2B, we used a between-subjects design to search for correspondence between familiarity and déjà vu; participants rated either degree of familiarity or déjà vu, but not both. In the déjà vu condition, déjà vu was not defined.

\section{Method}

Participants. Fifty Colorado State University undergraduates participated in Experiment 2A and 94 participated in Experiment 2B in exchange for course credit.

Materials. The study and test materials were identical to those in Experiment 1.

Procedure. The procedure was identical to that used in Experiment 1, but with the following exceptions. In Experiment 2A, participants were merely prompted to indicate the presence or absence of a déjà vu state following the first attempt at cued recall. They gave no ratings, and déjà vu was not defined for them. In Experiment 2B, ratings of déjà vu intensity, rather than yes-no responses, were used. Stimuli were counterbalanced across the similar and dissimilar conditions (rather than being randomly assigned), ${ }^{5}$ and participants were randomly assigned to either the familiarity condition or the déjà vu condition. In the familiarity condition, following the attempt at cued recall, participants were prompted to rate the familiarity of the test scene as in Experiment 1, but there was no reference to déjà vu. In the déjà vu condition, participants were instead prompted to rate their feeling of déjà vu following the attempt at cued recall. Déjà vu was not defined; participants were simply told to rely on their own definitions of déjà vu.

\section{Results and Discussion}

Experiment 2A. Even when the déjà vu question was not juxtaposed with familiarity and not defined for participants, the probability of a reported déjà vu state was greater when a test scene resembled an unrecallable studied scene $(M=.26)$ than when it did not $(M=.23)$ $\left[t_{\text {subjects }}(49)=2.25, S E=0.01, p=.029 ; t_{\text {items }}(117)=\right.$ 1.96, $S E=0.02, p=.05] .{ }^{6}$ These probabilities did not differ significantly across blocks. As in Experiment 1, among test items resembling studied scenes, the probability of déjà vu was higher in the presence of recall $(M=$ $.68)$ than in its absence $(M=.26)[t(49)=12.64, S E=$ $0.03, p<.001]$.

Experiment 2B. A 2 (similarity condition: similar vs. dissimilar to a studied scene) $\times 2$ (ratings condition: familiarity vs. déjà vu ratings) mixed ANOVA performed on ratings given in the absence of successful cued recall revealed three findings of interest (see Table 2). First, Experiment $2 \mathrm{~B}$ replicated the RWCR effect shown in Experiment 1 ; ratings were higher for test scenes resembling studied scenes than for test scenes not resembling studied scenes $\left[F_{\text {subjects }}(1,92)=58.38, M S_{\mathrm{e}}=0.18, p<.001, \eta_{\mathrm{p}}^{2}=.39\right.$; $\left.F_{\text {items }}(1,237)=28.40, M S_{\mathrm{e}}=1.27, p<.001, \eta_{\mathrm{p}}^{2}=.11\right]$.

Second, the magnitude of the RWCR effect did not differ as a function of whether familiarity or déjà vu ratings were given: There was no interaction $\left[F_{\text {subjects }}(1,92)=0.58\right.$, $M S_{\mathrm{e}}=0.18, p=.45 ; F_{\text {items }}(1,237)=0.69, M S_{\mathrm{e}}=1.27$, $p=.41]$. Thus, even when familiarity and déjà vu were not juxtaposed within the experiment, there was great cor-
Table 2

Mean Scene Familiarity and Déjà Vu Ratings Given in the Absence of Cued Recall

\begin{tabular}{|c|c|c|c|c|}
\hline \multirow[b]{3}{*}{ Experiment } & \multicolumn{4}{|c|}{ Test Scene Type } \\
\hline & \multicolumn{2}{|c|}{$\begin{array}{l}\text { Configurally } \\
\text { Similar } \\
\text { to a Studied } \\
\text { Scene }\end{array}$} & \multicolumn{2}{|c|}{$\begin{array}{c}\text { Configurally } \\
\text { Dissimilar } \\
\text { to a Studied } \\
\text { Scene }\end{array}$} \\
\hline & $M$ & $S D$ & $M$ & $S D$ \\
\hline $\begin{array}{l}1 \text { Familiarity ratings } \\
\text { (familiarity \& déjà vu juxtaposed) }\end{array}$ & 2.30 & 1.42 & 1.97 & 1.30 \\
\hline 2B Familiarity ratings & 3.00 & 1.24 & 2.48 & 1.19 \\
\hline 2B Déjà vu ratings & 2.36 & 1.46 & 1.93 & 1.23 \\
\hline
\end{tabular}

respondence between judging familiarity and judging the feeling of being in a déjà vu state with a scene. Both types of judgment gave rise to a comparably sized RWCR effect, even when déjà vu was not defined for participants.

Third, ratings were higher in the familiarity than in the déjà vu condition $\left[F(1,92)=5.34, M S_{\mathrm{e}}=3.11, p=.023\right.$, $\eta_{\mathrm{p}}^{2}=.06 ; F_{\text {items }}(1,237)=28.20, M S_{\mathrm{e}}=1.47, p<.001$, $\left.\eta_{\mathrm{p}}^{2}=.11\right]$. Participants were more conservative with their ratings in the déjà vu condition than in the familiarity condition, even when successful cued recall occurred: Ratings accompanied by recall were higher in the familiarity condition $(M=7.09)$ than in the déjà vu condition $(M=$ 6.19) $[t(92)=3.26, S E=0.28, p=.002]$. This may reflect a general reluctance to indicate feelings of déjà vu on the part of the participants, as prior survey research has shown that people only rarely experience déjà vu (Brown, 2003). However, despite this general conservative bias in the déjà vu condition, the magnitude of the RWCR effect remained the same as in the familiarity condition.

\section{GENERAL DISCUSSION}

The present results suggest that déjà vu is related to familiarity and that it can be elicited by similarity to a previous experience. When an experimentally novel scene resembled a previously viewed scene in configuration, increased familiarity with the novel scene occurred, even when the studied scene responsible for that increased familiarity could not be recalled. This RWCR effect (e.g., Cleary, 2004) appears to be related to déjà vu. First, configural similarity of a novel scene to a previously viewed but unrecalled scene increased the probability of a reported déjà vu state with the novel scene. Second, familiarity ratings were directly related to reported déjà vu states: Feelings of familiarity that accompanied reported déjà vu states were stronger than those that accompanied reported non-déjà vu states. Taken together, these results suggest that feelings of familiarity and feelings of déjà vu are subjectively similar.

Even when demand characteristics were reduced by eliminating the juxtaposition of familiarity and déjà vu questions, the familiarity brought on by configural resemblance appeared to be related to déjà vu. First, even when familiarity was not mentioned and déjà vu was not defined, configural resemblance increased the probability 
of a reported déjà vu state. Second, in a between-subjects design, both types of rating (familiarity and déjà vu) gave rise to an RWCR effect of comparable magnitude, even when déjà vu was not defined.

That déjà vu may be elicited by resemblance to a prior experience suggests that it can be stimulus driven. O'Connor and Moulin (2008) found that the chronic déjà vu experienced by a temporal-lobe-epileptic male occurred in the absence of any eliciting external stimuli, appearing instead to be produced by spontaneous neural activity related to the seizure disorder. Chronic déjà vu associated with seizures may indeed be internally driven, but this does not mean that déjà vu can never be stimulus driven, especially in nonepileptic participant populations. The fact that people report experiencing déjà vu more often with scenes than with any other stimulus type (Brown, 2004) supports the stimulus-driven hypothesis. Our study further supports it: People are more likely to report experiencing déjà vu with a novel situation that resembles one stored in memory.

Some may be concerned that the probability of reporting a déjà vu experience in our study was higher than might be expected, since survey research suggests that déjà vu is a relatively rare experience (Brown, 2003). We have three responses to this concern. First, our sample consists of the subpopulation with the highest frequency of reported déjà vu experiences: college-aged adults (Brown, 2004).

Second, even if dejà vu experiences are typically infrequent, there are circumstances that can increase the likelihood of a déjà vu experience, as in cases of certain kinds of temporal-lobe epilepsy (e.g., Bowles et al., 2007; O'Connor \& Moulin, 2008). In theory, if déjà vu can be stimulus driven, it should be possible to experimentally increase the likelihood of a déjà vu state by creating the necessary stimulus conditions. Still, even with our efforts to reduce demand characteristics, the probability of a reported déjà vu experience in the control (i.e., dissimilar) condition of the present study was not as close to zero as some might expect. Some potential explanations are that (1) merely having déjà vu on one's mind increases the likelihood of a déjà vu experience; (2) creating stimulus conditions that favor the elicitation of déjà vu drive up the overall inclination to report a déjà vu; (3) repeatedly engaging in the same types of test trials leads to a sense of having experienced a new test trial before; and (4) it is possible that there was some unintended resemblance between studied scenes and test scenes not intended to resemble studied scenes.

The fact that participants grew less conservative with their déjà vu responses as the experiment progressed supports the first three possibilities. However, none of these possibilities compromises our finding that similarity to a studied episode increases the probability of a déjà vu state.

Third, it is not yet known whether people experience familiarity in day-to-day life with the same frequency that they report it in the lab. It is possible that the experimental conditions for studying familiarity increase the likelihood of responding that something is familiar relative to familiarity's occurrence in day-to-day life. To our knowledge, no survey studies have examined the frequency of reported familiarity experiences or how this compares to déjà vu. This could, perhaps, be an avenue for future survey research.

What differentiates familiarity from déjà vu? Although we are unaware of any survey studies comparing familiarity and déjà vu, one aspect of our data suggests that participants do make at least one differentiation between the two: Participants were more conservative with their déjà vu responses than with their familiarity responses. This could indicate that participants viewed déjà vu as being a less probable experience than familiarity is.

Our data did not support the hypothesis that participants differentiate feelings of familiarity from feelings of déjà vu on the basis of the presence or absence of recall. Overall, there was high correspondence between judgments of déjà vu and judgments of familiarity, regardless of whether recall occurred. Whether participants rated degree of familiarity or déjà vu, they gave higher ratings in the presence of recall than in its absence. In this regard, participants did not treat déjà vu differently from familiarity; participants typically give higher familiarity ratings in the presence than in the absence of recall (Cleary, 2004). Although little is known about how recall relates to familiarity, it is conceivable that test items that elicited cued recall of their similar studied counterparts were also those highest in familiarity to the participants. Furthermore, participants received no feedback regarding whether they got the correct answer after a recall attempt. In the face of such uncertainty about whether they had recalled the correct episode responsible for the familiarity with a test scene, participants may have been inclined to base their feeling of déjà vu strictly on the level of familiarity of the test scene.

Interestingly, participants were more inclined to give déjà vu responses in the presence of recall when déjà vu was not experimentally defined. In a cross-experimental comparison between Experiment 1 and Experiment 2A, a 2 (definition condition: déjà vu defined vs. déjà vu not defined) $\times 2$ (recall status: presence vs. absence of recall) mixed ANOVA performed on the proportion of déjà vu states in the similar condition revealed a significant interaction $\left[F(1,122)=8.09, M S_{\mathrm{e}}=0.03, p=.005\right]$, such that the tendency to give more déjà vu responses in the presence than in the absence of recall was greater when déjà vu was not defined. This suggests that participants' preexperimental notions about déjà vu may make them more inclined to equate déjà vu with standard feelings of familiarity.

Questionnaire data were consistent with this suggestion. We asked an independent group of 92 participants to define déjà vu. Of them, 73 defined it merely as a sense of having experienced something before; only 6 defined it as the sense of having experienced something before in the face of evidence that the situation was new (the remaining 13 had a conglomeration of ideas about déjà vu that did not fit these two categories). If participants generally preexperimentally defined déjà vu in the same way that 
they defined familiarity, it would explain the great correspondence between familiarity and déjà vu judgments in the present study.

Whereas the present study supports the hypothesis that familiarity and déjà vu are related, future research might aim to better understand whether and how familiarity and déjà vu differ. Survey research comparing déjà vu and familiarity experiences in day-to-day life could help in this endeavor. Future experimental research should aim to determine whether other factors (besides degree of conservativism) experimentally differentiate déjà vu from familiarity. Such research might focus on instructions. With more careful instructions regarding déjà vu states, it may be possible to produce a pattern of lower feelings of déjà vu in the presence of recall than in its absence. A trade-off of doing so might be the creation of demand characteristics that would prevent an investigation of participants' preexperimental notions of déjà vu. However, until familiarity and déjà vu can be dissociated in the laboratory on dimensions other than overall conservativism, it remains possible that they are the same underlying construct.

\section{AUTHOR NOTE}

This study was supported by NSF Grant BCS-0638486 to the first author. We thank Mary Lee, James Lee, Amanda Pala, Jessie Savre, Carly Smith, and Danielle Vannucci for assistance with data collection. Correspondence concerning this article should be addressed to A. M. Cleary, Department of Psychology, Colorado State University, 1876 Campus Delivery, Fort Collins, CO 80523-1876 (e-mail: anne.cleary@, colostate.edu)

\section{REFERENCES}

Bowles, B., Crupi, C., Mirsattari, S. M., Pigott, S. E., Parrent, A. G., Pruessner, J. C., ET AL. (2007). Impaired familiarity with preserved recollection after anterior temporal-lobe resection that spares the hippocampus. Proceedings of the National Academy of Sciences, 104, 16382-16387. doi:10.1073/pnas.0705273104

Brown, A. S. (2003). A review of the déjà vu experience. Psychological Bulletin, 129, 394-413. doi:10.1037/0033-2909.129.3.394

Brown, A. S. (2004). The déjà vu experience. New York: Psychology Press.

Brown, A. S., \& Marsh, E. J. (2008). Evoking false beliefs about autobiographical experience. Psychonomic Bulletin \& Review, 15, 186190. doi:10.3758/PBR.15.1.186

Cleary, A. M. (2004). Orthography, phonology, and meaning: Word features that give rise to feelings of familiarity in recognition. Psychonomic Bulletin \& Review, 11, 446-451.
Cleary, A. M. (2008). Recognition memory, familiarity, and déjà vu experiences. Current Directions in Psychological Science, 17, 353357. doi: $10.1111 / j .1467-8721.2008 .00605 . x$

MANDLER, G. (2008). Familiarity breeds attempts: A critical review of dual-process theories of recognition. Perspectives on Psychological Science, 3, 390-399. doi:10.1111/j.1745-6924.2008.00087.x

O'Connor, A. R., \& Moulin, C. J. A. (2008). The persistence of erroneous familiarity in an epileptic male: Challenging perceptual theories of déjà vu activation. Brain \& Cognition, 68, 144-147.

van Diepen, P. M. J., \& De Graef, P. (1994). Line-drawing library and software toolbox (Psychology Rep. No. 165). Leuven, Belgium: University of Leuven, Laboratory of Experimental Psychology.

Wallisch, P. (2007, May). The déjà vu experience as episodic source memory failure. Paper presented at the 79th Annual Meeting of the Midwestern Psychological Association, Chicago.

\section{NOTES}

1. To ensure that configural resemblance indeed triggers familiaritybased recognition (and not just recall), it is important to examine judgments given in the absence of recall.

2. Some scenes used with permission from "Line-Drawing Library and Software Toolbox," by P. M. J. van Diepen and P. De Graef, 1994, (Psychology Rep. No. 165). Copyright 1994 by the University of Leuven, Belgium.

3. Some might be concerned that participants are recalling the studied scene itself but fail to recollect its name. To address this, we ran a variation of Experiment 1, in which the test contained the actually studied pictures without their names. The participants' task was merely to name each picture. Performance was at ceiling. The mean identification rate was .99. This suggests that, if a participant could recollect a studied picture in response to a test cue, he or she would almost always be able to provide its name.

4. In Experiment 1, 22 participants indicated no déjà vu state at all in at least one of the conditions of interest and therefore could not be included in an analysis of how ratings varied as a function of déjà vu state. The remaining 52 participants still showed the RWCR effect: These participants gave higher ratings to scenes that resembled unidentified studied scenes $(M=2.54, S D=1.45)$ than to scenes that did not resemble studied scenes $(M=2.17, S D=1.34)\left[t_{\text {subjects }}(51)=4.99, S E=\right.$ $0.07, p<.001 d=0.70]$.

5. Due to the way that the counterbalancing was done in Experiment 2B, stimuli were not randomly assigned to blocks; therefore, a meaningful analysis of performance across blocks could not be performed.

6. For two test items, all but 1 participant recalled the corresponding studied scene. Because this left only one opportunity for a potential déjà vu response in the studied condition for these two items (analogous to having only one item for a participant in a participant analysis), they were excluded from item analysis.

(Manuscript received October 10, 2008; revision accepted for publication August 22, 2009.) 